\title{
Magnetic and Electrical Transport Properties of Vanadium Telluride
}

\author{
C. Prasad, R. Shanker, and R. A. Singh \\ Department of Physics, University of Gorakhpur, Gorakhpur-273001, India
}

Z. Naturforsch. 35 a, $701-703$ (1980) ; received March 30, 1980

\begin{abstract}
Measurements of the magnetic susceptibility of a powdered sample of VTe in the temperature range $90-700 \mathrm{~K}$, and of the a.c. electrical conductivity $(\sigma)$, thermoelectric power $(\theta)$ and dielectric constant $\left(\varepsilon^{\prime}\right)$ of pressed pellets of the compound in the temperature range $300-1100 \mathrm{~K}$ are reported. The compound is found to be antiferromagnetic with Neel temperature $420 \pm 5 \mathrm{~K}$. The effective paramagnetic moment and paramagnetic Curie temperature are found to be $1.6 \mu_{\mathrm{B}}$ and $-250 \mathrm{~K}$, respectively. The dependence of $\sigma, \theta$ and $\varepsilon^{\prime}$ on temperature shows no anomaly at the Neel temperature and is indicative of the metallic nature of the compound.
\end{abstract}

\section{Introduction}

Considerable attention has been centred on the study and utilisation of transition metal compounds, of which the Chalcogenides TX (where $\mathrm{T}=$ Transition metal and $\mathrm{X}=\mathrm{S}$, Se or $\mathrm{Te}$ ) form a special group of magnetically ordered compounds $[1-5]$. VTe is one of the Chalcogenides which was not yet thoroughly investigated. It has the Nickel-Arsenide type crystal structure, which is composed of a closepacked-hexagonal anion sublattice with cations in the octahedral interstices forming a simple hexagonal sublattice. Along the $c$-axis the octahedral sites share common faces both above and below, so that the cations are arranged in linear chains with optimum conditions for cation - cation interactions, each octahedral site cation seeing a trigonal ligand field. The lattice specifications are $a=3.813 \AA$ and $c / a=$ 1.609 .

The present paper reports on measurement of the magnetic susceptibility, a.c. electrical conductivity, thermoelectric power and static dielectric constant of $\mathrm{VTe}$ in a wide temperature range.

\section{Experimental Details}

Our sample (purity 99.99\%), procured from Messers Ventron, Alfa products, USA, was in powder form and we were unable to prepare single crystals due to experimental limitations. We, therefore, have used pressed pellets for the electrical conductivity, thermoelectric power and dielectric constant measurements.

Reprint requests to Dr. R. A. Singh, Department of Physics, University of Gorakhpur, Gorakhpur-273001, India.
The magnetic susceptibility $\left(\chi_{\mathrm{g}}\right)$ was measured on a finely powdered sample in the temperature range $90-300 \mathrm{~K}$ with the help of an electrodynamically controlled Curie type magnetic balance having a photoelectric detecting system $[6,7] . \chi_{g}$ of the sample in the temperature range $300-700 \mathrm{~K}$ was measured using a sensitive balance with the finely powdered sample suspended from one of the arms and hanging inside a cylindrical furnace kept between the pole-pieces of a strong electromagnet with a vertical field gradient [8]. The temperature in the vicinity of the sample was measured with a chromelalumel thermocouple with an accuracy of $1 \mathrm{~K}$. The susceptibility measurements were done at a field strength of $1.8 \mathrm{kOe}$. For the measurement of the electrical conductivity, thermoelectric power and dielectric constant the powder specimen was pelletized at $\approx 5 \times 10^{6} \mathrm{~g} / \mathrm{cm}^{2}$ pressure. The pellet was mounted in a stainless steel sample holder, similar to that of Shahi and Chandra [9], between platinum electrodes electrically insulated from the sample holder. The a.c. conductivity was measured with a Systronic conductivity meter type 302 (INDIA) operating at $2 \mathrm{kHz}$, the thermoelectric power with an OSAW (INDIA) Vernier potentiometer model 30071, and the dielectric constant with an LCR bridge model Ruttonsha-Simpson 901 I (INDIA) working at $1 \mathrm{kHz}$.

\section{Results and Discussion}

Figure 1 shows the variation of $\chi_{g}$ and $\chi_{g}{ }^{-1}$ with temperature. The susceptibility below $345 \mathrm{~K}$ is large and found to be field dependent. Above $345 \mathrm{~K}, \chi_{\mathrm{g}}$ increases with temperature and a maximum occurs

0340-4811 / $80 / 0700-0701 \$ 01.00 / 0$. - Please order a reprint rather than making your own copy. 


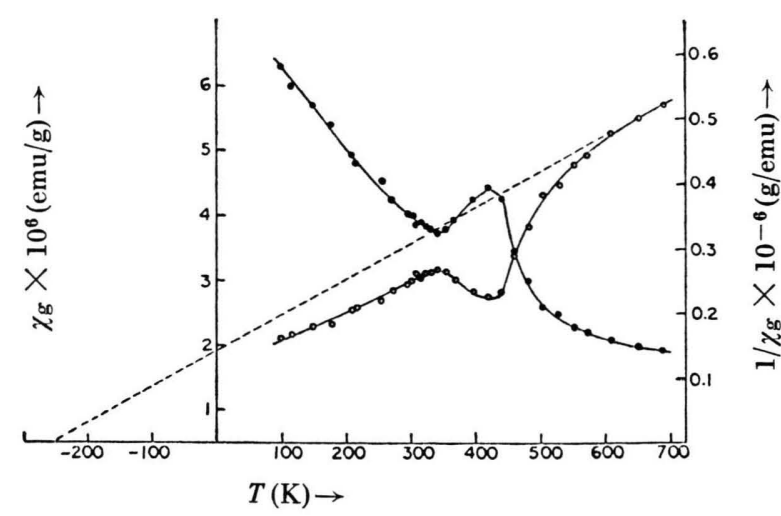

Fig. 1. Variation of $\chi_{\mathrm{g}}$ and $\chi_{\mathrm{g}}{ }^{-1}$ with temperature for a powdered sample of Vanadium Telluride.

at the Neel temperature $T_{\mathrm{N}}=420 \pm 5 \mathrm{~K}$ indicating that antiferromagnetic ordering takes place. From the figure, the effective paramagnetic moment, $p_{\text {eff }}$, and paramagnetic Curie temperature, $H_{\mathrm{c}}$, are found to be $1.6 \mu_{\mathrm{B}}$ and $-250 \mathrm{~K}$, respectively.

Figure 2 shows variation of the a.c. electrical conductivity $(\sigma)$ with temperature. No anomaly was observed in the conductivity at the transition temperature. The decrease of $\sigma$ with temperature suggests a metallic nature of the compound. The metallic conductivity may be thought of as a case of single band conduction with the partially filled $3 \mathrm{~d}$-band of the $\mathrm{V}^{++}$ion forming the conduction band and the $\mathrm{Te}^{--}$ ion acquiring inert gas structure. However, its binding is not completely ionic, but intermediate between ionic and metallic, as is evidenced from the electrical conductivity which is larger than expected for an ionic compound [1]. The metallic character is

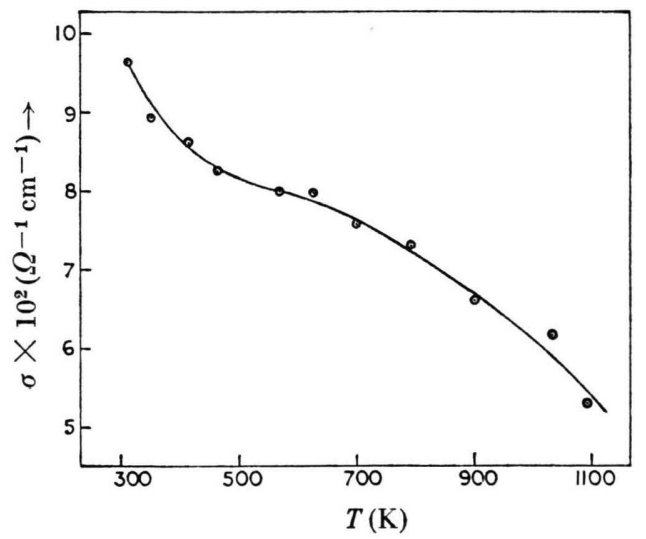

Fig. 2. Variation of $\sigma$ with temperature for a Vanadium Telluride pellet. further supported by the metallic lustre, hardness and brittleness of VTe. In NiAs type compounds, the presence of an interstitial cation forces $c / a$ to be less than 1.63. Since $c / a=1.609$ for VTe, metallic d-band conduction can occur via the interstitials [10]. But, the presence of interstitials does not solely determine the origin of the metallic conductivity. In fact, Goldschmidt [11] has pointed out that due to polarisation phenomena, arising from the mutual interaction of the cation and the anion, metallic character will be developed in substances of $\mathrm{NiAs}$ structure, where a transition metal of relatively small size is combined with a large, readily polarisable element. Also, since the conduction is due to a narrow d-band, the conduction electrons are partly localized [12] and hence the conductivity is less than for pure metals.

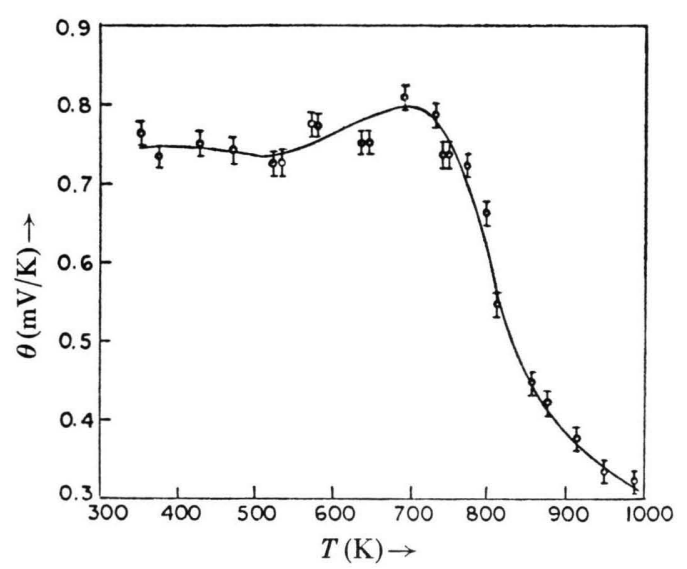

Fig. 3. Variation of $\theta$ with temperature for a Vanadium Telluride pellet.

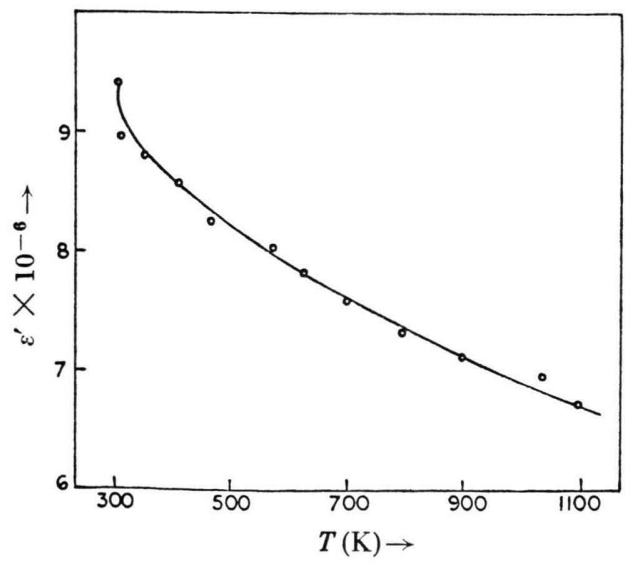

Fig. 4. Variation of $\varepsilon^{\prime}$ with temperature for a Vanadium Telluride pellet. 
The variation of the thermoelectric power $(\theta)$ with temperature is shown in Figure 3. No anomaly was found at the magnetic transition temperature $(420 \pm 5 \mathrm{~K})$ though a peak was observed at about $700 \mathrm{~K}$ followed by a rather rapid decrease to approximately one third of the peak value. The positive thermoelectric power suggests that electrons are the majority charge carriers. This can be confirmed by a Hall-experiment. Figure 4 shows the variation of the static dielectric constant $\left(\varepsilon^{\prime}\right)$ with temperature. It decreases with increasing temperature. The large

[1] K. Adachi, J. Phys. Soc. Japan 16, 2187 (1961).

[2] G. G. Dvoryankina and Z. G. Pinsker, Kristallografiya 7, 458 (1962).

[3] G. Zanmarchi, J. Phys. Chem. Sol. 28, 2123 (1967).

[4] M. M. Srivastava and O. N. Srivastava, Acta Crystallogr. A (Denmark) A 31, 82 (1975).

[5] T. Ito, K. Ito, and M. Oka, Japan. J. Appl. Phys. 17, 371 (1978).

[6] D. Guha Thakurta and D. Mukhopadhyay, Indian J. Phys. 40, 69 (1966).

[7] D. Ghosh, D. Phil Thesis Calcutta University 1970. values of the dielectric constant are also an indication of the metallic nature of the compound.

\section{Acknowledgements}

The authors are thankful to the authorities of Indian Association for the Cultivation of Science, Calcutta, India for providing facilities for low temperature susceptibility measurements. They are thankful to C.S.I.R. New Delhi for financial assistance. Thanks are also due to Professor Nitish K. Sanyal for the encouragement.

[8] L. F. Bates, Modern Magnetism, University Press, Cambridge 1951.

[9] K. Shahi and S. Chandra, Phys. Stat. Sol. 28 a, 653 (1975).

[10] J. B. Goodenough, Magnetism and the Chemical Bond, John Wiley, New York 1966.

[11] P. R. Ray and D. N. Sen, J. Indian Chem. Soc. 25, 209 (1948).

[12] J. P. Suchet, Electrical Conduction in Solid Materials, Pergamon Press, London 1975. 